\title{
多模态目标的联合压缩跟踪
}

\author{
唐艳平 1 ，张灿龙 ${ }^{2,3)^{*}}$ ，李燕茹 ${ }^{2)}$ ，李志㸝 2,3 ) \\ ${ }^{1)}$ (桂林电子科技大学计算机与信息安全学院 桂林 541004) \\ 2) (广西师范大学广西多源信息挖掘与安全重点实验室 桂林 541004) \\ 3) (广西区域多源信息集成与智能处理协同创新中心 桂林 541004) \\ (zcltyp@163.com)
}

\begin{abstract}
摘 要: 针对多模态目标跟踪中大多仅考虑单个图像的异种特征融合或不同模态图像的同种特征融合, 为了使得这 两者间能自然集成, 提出基于联合压缩感知的多模态目标统一跟踪方法. 通过将多模态跟踪问题转化为多重 $\ell_{2}$-范数 不等式约束下的多 $\ell_{1}$-范数联合最小化问题, 并设计了能求解该联合最小化问题的特定增广拉格朗日乘子算法, 从而 实现快速而精准的目标跟踪，可同时处理来自同一图像或不同模态图像中的多种不同特征间的融合，并能自由地添 加或删除特征. 此外, 还提出了基于稀疏集中度指标的目标模板协同更新方案, 来篮选出表现最优的目标模板. 在 DCU, OTCBVS, BEPMDS, OTB50 和 VOT-TIR 等数据集上采用逐帧跟踪的方法进行实验，结果表明在跟踪精度、成 功率以及速度 3 个评价指标上, 文中方法的平均性能分别达到了 $0.96,0.91$ 和 3.48.
\end{abstract}

关键词: 多模态跟踪; 压缩感知; 增广拉格朗日乘子法

中图法分类号: TP391.41 DOI: 10.3724/SP.J.1089.2020.17813

\section{Joint Compressive Tracking for Multimodal Target}

\author{
Tang Yanping ${ }^{1)}$, Zhang Canlong ${ }^{2,3)^{*}}$, Li Yanru' ${ }^{2)}$, and Li Zhixin ${ }^{2,3)}$ \\ 1) (School of Computer Science and Information Security, Guilin University of Electronic Technology, Guilin 541004) \\ 2) (Guangxi Key Laboratory of Multi-Source Information Mining and Security, Guangxi Normal University, Guilin 541004) \\ ${ }^{3)}$ (Guangxi Collaborative Innovation Center of Multi-Source Information Integration and Intelligent Processing, Guilin 541004)
}

\begin{abstract}
Multimodal tracking is a challenging task. Most of the existing methods only consider the fusion of different features from single image or the fusion of identical feature from different modal images. In order to integrate them naturally, a unified multimodal tracking framework based on joint compressive sensing is proposed in this paper. Our framework can integrate different features extracted from single image or different modal images, and provide the flexibility to arbitrarily add or remove feature. We formulate the multimodal tracking as a joint minimization problem of multiple $\ell_{1}$-norms with inequality constraint of multiple $\ell_{2}$-norms, and derive a customized augmented Lagrange multiplier algorithm to solve the minimization problem, so that efficient tracking with both low computational burden and high accuracy. Besides, a collaborative template update scheme induced by sparsity concentration index is developed to screen out the best templates throughout the tracking procedure. The experiments are carried out on DCU, OTCBVS, BEPMDS, OTB50 and VOT-TIR image datasets by frame tracking method, and the experimental results show the average tracking accuracy, success rate and speed of our method is $0.96,0.91$ and 3.48 respectively.

收稿日期：2019-05-27；修回日期：2020-01-16。基金项目：国家自然科学基金(61866004，61663004，61966004，61962007, 61751213); 广西自然科学基金(2018GXNSFDA281009, 2017GXNSFAA198365, 2019GXNSFDA245018, 2018GXNSFDA294001); 广西 “八桂学者”创新研究团队. 唐艳平(1977一), 女, 硕士, 讲师, 主要研究方向为图像处理与目标跟踪; 张灿龙(1975一), 男, 博士, 教授, 博士生导师, CCF 会员, 论文通讯作者, 主要研究方向为图像理解与机器学习; 李燕茹(1994一), 女, 硕士研究生, 主要研究方向 为目标跟踪; 李志欣 $(1971-)$, 男, 博士, 教授, 博士生导师, CCF 会员, 主要研究方向为图像理解与机器学习.
\end{abstract}


Key words: multimodal tracking; compressive sensing; augmented Lagrange multiplier (ALM)

目标跟踪是视频监控、人机交互和车辆导航等 应用的基础. 近 20 年来, 人们提出了大量的跟踪 方法, 这些方法可大致分为单模态 ${ }^{[1-6]}$ 和多模态 2 大类 ${ }^{[7-17]}$. 与单模态跟踪相比, 多模态融合跟踪利 用了不同模态的互补性, 因而能够更好地描述复 杂情况下的目标. 单模态跟踪方法述超出了本文 的范围, 详见文献[18-19].

在多模态跟踪中, 特征可能来自单个图像, 也 可能来自多模态图像，因此可以进一步将多模态 跟踪方法分为基于单图像多特征的方法 ${ }^{[7-11]}$ 和基 于单特征多图像的跟踪方法 ${ }^{[12-17]}$. 前者如利用局 部方向核描述符和颜色直方图来表示目标的融合 跟踪方法 ${ }^{[7]}$; 用于鲁棒特征级融合跟踪的联合稀 疏表示模型 ${ }^{[8]}$, 它可以动态地防止不可靠特征的融 合; 基于残差神经网络的 3 种端到端的融合跟踪框 架 ${ }^{[9]}$, 分别为像素级、特征级和响应级融合; 采用 哈希方法将不同的特征融合成紧致的二元特征的 融合跟踪方法 ${ }^{[10]}$; 以及利用多特征通道进行自适 应加权的在线跟踪算法 ${ }^{[11]}$.

不同模态的图像组合是很重要的, 因为每种 模态图像都提供了相异而互补的场景信息, 其中 可见光和红外图像是多模态组合中最活跃的研究 课题. 红外传感器可以检测出场景中物体产生的 热能差异, 因与光照无关, 使其在弱光照条件下比 可见光传感器更有效, 但红外图像不能提供颜色 和纹理特征. 另一方面, 可见光传感器对场景中的 温度差异不敏感，当物体处于热交叉时，只要场景 照明良好, 物体具有与背景不同的颜色特征, 则可 见光传感器通常比红外传感器更有效. 因此, 两者 的组合可以弥补各自的弱点, 跟踪器的性能要优 于单一传感器. 目前, 已有大量的红外-可见光目 标跟踪算法, 如基于全卷积孪生网络的彩色-红外 融合跟踪方法 ${ }^{[12]}$; 通过使用二阶空间直方图来整 合多种不同的模态，提出的统一的多模态跟踪框 架 ${ }^{13]}$ ，可自由地增加或删除模态; 基于模糊区域 动态图像融合的多角色均值漂移跟踪方法 ${ }^{[14]}$; 鉴 于均值漂移是一种局部确定性搜索方法, 很容易 陷入局部最小值, 所提出的先跟踪后融合的红外可见光目标跟踪方法 ${ }^{[15]}$, 利用改进的粒子滤波和 模板匹配算法分别对可见光目标和红外目标进行 跟踪, 并对其跟踪结果进行配准和融合. 但是当红 外光和可见光跟踪结果中的任何一个很差时, 导
致最终的跟踪结果也可能非常糟糕. 还有在贝叶 斯框架下基于压缩时空卡尔曼滤波的融合跟踪算 法 $^{[16]}$, 它允许集成独立的特征; 然而, 它的贝叶斯 分类需要大量的样本来获得足够的准确性, 这就 大大增加了计算量. 基于联合稀疏表示的红外可 见目标跟踪方法 ${ }^{[17]}$, 由联合稀疏表示推导而来的 距离度量函数被用来构造粒子滤波的似然函数, 从而使色谱和热谱图像能融合到目标跟踪中. 其 中, 红外或可见光图像只用了一个目标模板, 其稀 疏分解是在目标候选模板和细碎模板级联的基础 上进行的, 该方法只需解一个 $\ell_{1}$-范数相关的最小 化问题, 所以时间成本大幅度降低. 但是, 只使用 一个目标模板会失去模板的多样性, 从而降低跟 踪器的鲁棒性. 如果直接使用 $\ell_{1}$ 跟踪器, 对于求解 高维 $\ell_{1}$-最小化问题的计算量会很大.

上述 2 类多模态跟踪方法各有各的特点, 这使 得其难以自然地集成, 本文将解决这个问题. 相关 研究中, 多任务联合稀疏表示和分类算法 ${ }^{[20]}$ 给出 了可视化特征组合的核扩展; 基于压缩感知的跟 踪算法 ${ }^{[21]}$ 给出了 $\ell_{1}$ 跟踪问题的压缩表示形式, 并 设计了一种正交匹配追踪方法求解该问题, 从而 大大地提速了 $\ell_{1}$ 跟踪; 通过采用双增广拉格朗日 乘子(dual augmented Lagrange multiplier, DALM) 方法 ${ }^{[22]}$ 开发的一种非常快速的数值求解器来解决 $\ell_{1}$ 范数相关的最小化问题. 受此启发, 本文提出一 种基于联合压缩感知的统一多模态跟踪框架. 如 果把每个特征看做一种模态, 而每个模态的候选 目标被分解为对应模态的目标模板和细碎模板的 稀疏线性组合, 那么融合跟踪问题就可转化为求 解所有模态的候选目标与其相应的目标模板之间 的最小联合重构误差, 并通过解由多个 $\ell_{1,2}$ 混合范 数组合的最小化问题来实现.

\section{1 相关理论}

\section{1 基于压缩传感的 $\ell_{1}$ 跟踪}

假设 $\boldsymbol{a} \in \mathbb{R}^{N}$ 是目标系数向量, $\boldsymbol{e} \in \mathbb{R}^{d_{0}}$ 是普通 系数向量, $\boldsymbol{T}=\left[\boldsymbol{t}_{1}, \cdots, \boldsymbol{t}_{N}\right] \in \mathbb{R}^{d_{0} \times N}$ 是由 $N$ 个预先获得 的目标模板 $\boldsymbol{t}_{n} \in \mathbb{R}^{d_{0}}, \boldsymbol{E}=[\boldsymbol{I},-\boldsymbol{I}] \in \mathbb{R}^{d_{0} \times 2 d_{0}}$ 是普通模 板集; 其中, $\boldsymbol{I}$ 是 $d_{0} \times d_{0}$ 恒等矩阵, $\boldsymbol{b} \in \mathbb{R}^{d_{0}}$ 是目标 候选模板. $\ell_{1}$ 跟踪器采用正约束恢复稀疏系数 $\boldsymbol{x}$, 即 


$$
\min _{\boldsymbol{x}}\|\boldsymbol{x}\|_{1} \quad \text { s.t. } \quad \boldsymbol{b}=\boldsymbol{A} \boldsymbol{x}, \boldsymbol{x} \succeq 0
$$

其中, $\boldsymbol{A}=\left[\begin{array}{ll}\boldsymbol{T} & \boldsymbol{E}\end{array}\right]$ 是目标模板和带有 $\boldsymbol{x}=\left[\begin{array}{ll}\boldsymbol{a} & \boldsymbol{e}\end{array}\right]^{\mathrm{T}}$ 的普 通模板的组合. 注意, $\boldsymbol{t}_{n}$ 是一个向量, 其条目是带有 $d_{0}$ 像素的下采样目标修补程序的像素值, $\boldsymbol{b}$ 也是如 此. 由于式(1)的计算量很大, 因此 $d_{0}$ 不能太大, 但 $d_{0}$ 也不能太小, 否则会使跟踪精度的急剧下降.

本文在使用压缩传感时并不存在这种两难情况. 众所周知, 如果测量矩阵 $\boldsymbol{\Phi} \in \mathbb{R}^{d \times d_{0}}$ 遵循受限压缩感 知测量矩阵之有限等距性质, 则稀疏信号 $\boldsymbol{x}$ 可以从

$$
\min _{\boldsymbol{x}}\|\boldsymbol{x}\|_{1} \quad \text { s.t. } \quad \boldsymbol{\Phi} \boldsymbol{b}=\boldsymbol{\Phi} \boldsymbol{A} \boldsymbol{x}, \boldsymbol{x} \succeq 0
$$

中恢复到很高的概率. 其中, 测量矩阵的典型选择 就是随机高斯矩阵, 即 $\boldsymbol{\Phi}_{i, j} \sim \mathcal{N}(0,1)$. 随机矩阵将数 据从高维图像空间 $\boldsymbol{b} \in \mathbb{R}^{d_{0}}$ 投影到低维压缩空间 $\boldsymbol{\Phi} \boldsymbol{b} \in \mathbb{R}^{d}$ ，其中 $d \ll d_{0}$. 式(2)的计算复杂性依赖于 $d$, 因此降维后其求解速度大大加快.

在文献[21]中，采用自定义正交匹配追踪算法 求解式(2), 然后用 $r=\|\boldsymbol{\Phi} \boldsymbol{b}-\boldsymbol{\Phi} \boldsymbol{T} \boldsymbol{a}\|_{2}$ 求出残差. 最后, 这种观测的可能性被更新为 $l=\exp (-k \cdot r)$, 其中 $k$ 是 一个预先设定的正常数.

\section{2 用 DALM 解 $\ell_{1}$ 最小化}

ALM 是现有的求解式(2)中 $\ell_{1}$ 极小化问题的最 有效的方法. ALM算法有 2 个版本: 原算法和对偶算 法, 每次迭代计算过程的复杂度分别是 $O\left(\left(N+2 d_{0}\right)^{2}\right)$ 和 $O\left(N+2 d_{0}\right)$. 显然, 在 $\ell_{1}$ 跟踪问题 中, DALM 应该是首选的, 因为 $\left(N+2 d_{0}\right)$ 列的字典远 大于 $d$ 行的字典. 为简单起见，可将问题式(2)重写为

$$
\min _{\boldsymbol{x}}\|\boldsymbol{x}\|_{1} \quad \text { s.t. } \quad \boldsymbol{c}=\boldsymbol{B} \boldsymbol{x}, \boldsymbol{x} \succeq 0
$$

其中, $\boldsymbol{B}=\boldsymbol{\Phi} \boldsymbol{A} \in \mathbb{R}^{d \times\left(N+2 d_{0}\right)}, \boldsymbol{c}=\boldsymbol{\Phi} \boldsymbol{b} \in \mathbb{R}^{d}$, 式(3)的 对偶问题为

$$
\min _{\boldsymbol{y}} \boldsymbol{c}^{\mathrm{T}} \boldsymbol{y} \quad \text { s.t. } \quad \boldsymbol{B}^{\mathrm{T}} \boldsymbol{y} \in \boldsymbol{C}
$$

其中， $\boldsymbol{C}=\left\{\boldsymbol{\theta} \in \mathbb{R}^{N+2 d_{0}}: \boldsymbol{\theta} \leqslant \mathbf{1}\right\} ，$ 相关的增广拉格朗
日函数为

$$
\min _{\boldsymbol{y}, \boldsymbol{z}}-\boldsymbol{c}^{\mathrm{T}} \boldsymbol{y}-\boldsymbol{x}^{\mathrm{T}}\left(\boldsymbol{z}-\boldsymbol{B}^{\mathrm{T}} \boldsymbol{y}\right)-\frac{\beta}{2}\left\|\boldsymbol{z}-\boldsymbol{B}^{\mathrm{T}} \boldsymbol{y}\right\|_{2}^{2}, \quad \boldsymbol{z} \in \boldsymbol{C}
$$

其中, $\beta>0$ 为一个成本参数, $\boldsymbol{x} \in \mathbb{R}^{N+2 d_{0}}$ 是拉格朗 日乘子(即原始变量). 由于很难同时解决上述问题, 因此本文采用了一种交替策略, 即在保持其他变量 不变的同时, 迭代最小化代价函数.

先给定 $\left(\boldsymbol{x}_{k}, \boldsymbol{y}_{k}\right)$ ，则式(5)关于 $\boldsymbol{z}$ 的最小值 $z_{k+1}$ 为

$$
\boldsymbol{z}_{k+1}=\mathcal{P}_{\boldsymbol{C}}\left(\boldsymbol{B}^{\mathrm{T}} \boldsymbol{y}_{k}+\boldsymbol{x}_{k} / \beta\right)
$$

其中, $\mathcal{P}_{\boldsymbol{C}}$ 表示在 $\boldsymbol{C}$ 上的投影算子; 再给定 $\left(\boldsymbol{x}_{k}, \boldsymbol{z}_{k+1}\right)$, 则式(5)关于 $\boldsymbol{y}$ 的最小值 $\boldsymbol{y}_{k+1}$ 为

$$
\boldsymbol{y}_{k+1}=\left(\boldsymbol{B} \boldsymbol{B}^{\mathrm{T}}\right)^{-1}\left(\boldsymbol{B} \boldsymbol{z}_{k+1}-\left(\boldsymbol{B} \boldsymbol{x}_{k}-\boldsymbol{c}\right) / \beta\right)
$$

假设 $\boldsymbol{B} \boldsymbol{B}^{\mathrm{T}}$ 是可逆的, 给定 $\left(\boldsymbol{y}_{k+1}, z_{k+1}\right)$ ，则

$$
\boldsymbol{x}_{k+1}=\boldsymbol{x}_{k}-\beta\left(\boldsymbol{z}_{k+1}-\boldsymbol{B}^{\mathrm{T}} \boldsymbol{y}_{k+1}\right)
$$

给定 $c$ 和 $B$, 初始化 $x_{1}=0, y_{1}=0$, 使用 DALM 算法迭代运行式(6) 式(8), 直到它收玫.

\section{2 联合压缩跟踪}

图 1 以可见光灰度、红外灰度和可见光局部二 值模式(local binary pattern, LBP)相结合为例, 展 示了所提出的多模态跟踪框架的工作机理. 首先 给定采样状态, 从当前帧中裁剪出具有不同特征 表示的多个目标候选块, 并通过随机高斯度量矩 阵对每个块进行重构和压缩到一个低维向量; 然 后, 将跟踪问题转化为寻找一个最优稀疏系数向 量, 使得所有补丁与相应压缩目标模板之间的联 合重构误差最小; 将其作为重构误差的函数计算 观测概率; 最后, 通过所有采样状态的观测概率, 以均方误差的方式估计目标状态.

假设有 $J$ 个不同的模态和 $M$ 个采样状态，那么 每个采样状态 $S_{i}$ 将对应于 $J$ 个具有不同模态的目 标图像. 也就是说, 在任何时刻 $t$, 所有已配准好

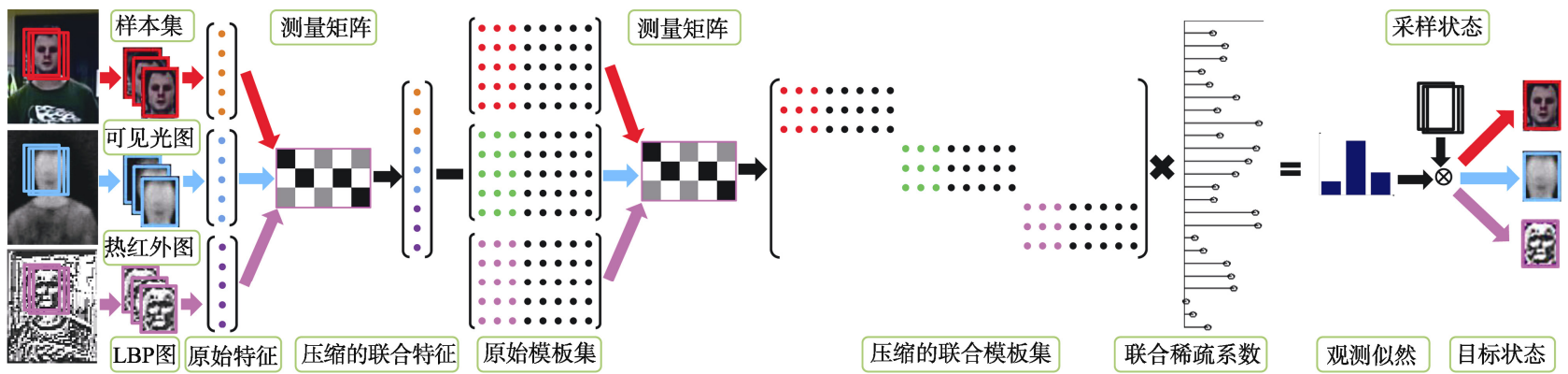

图 1 多模态融合跟踪方案示意图 
的具有不同模态的 $J$ 目标块都应该共享相同的运 动状态, 因为它们仅是使用不同的模态来描述同 一个真实的对象. 在本文的跟踪框架中, 状态变量 (即采样状态) 是由仿射变换参数 $\left(x, y, s_{x}, s_{y}, \theta, \phi\right)$ 建 模的, 其中 $(x, y)$ 是二维的平移参数, $\left(s_{x}, s_{y}, \theta, \phi\right)$ 是变形参数. $\left(s_{x}, s_{y}\right), \theta$ 和 $\phi$ 分别表示对对象的缩 放、旋转和错切. 通过以 $S_{i}$ 为参数应用仿射变换从 图像中裁剪出感兴趣的区域, 并将其规范化为与 图库中目标模板相同的大小. 采用多元零均值高 斯随机分布来模拟 2 个连续帧之间的状态转换.

\section{1 多模态联合压缩表示}

对于第 $j$ 个模态, 假设 $a^{j} \in \mathbb{R}^{N}$ 是它的目标系 数向量, $\boldsymbol{e}^{j} \in \mathbb{R}^{2 d_{0}}$ 是它的细碎系数向量, $\boldsymbol{b}_{i}^{j} \in \mathbb{R}^{d_{0}}$ 是它的第 $i$ 个候选目标, $\boldsymbol{T}^{j}=\left[\boldsymbol{t}_{1}^{j}, \cdots, \boldsymbol{t}_{N}^{j}\right] \in \mathbb{R}^{d_{0} \times N}$ 是 $N$ 个预先得到的目标模板, $\boldsymbol{E}^{j}=[\boldsymbol{I},-\boldsymbol{I}] \in \mathbb{R}^{d_{0} \times 2 d_{0}}$ 是 细碎模板集. 实际上, 每个模态的目标模板数目可 能是不同的, 甚至每个模态的模板大小也不同, 设 置 $N$ 和 $d_{0}$ 只是为了简化. 下面, 来考察 $J$-模态线 性压缩表示问题

$$
\boldsymbol{\Phi} \boldsymbol{b}_{i}^{j}=\boldsymbol{\Phi} \boldsymbol{A}^{j} \boldsymbol{x}^{j}, \quad j=1, \cdots, J
$$

其中, $\boldsymbol{A}^{j}=\left[\boldsymbol{T}^{j}, \boldsymbol{E}^{j}\right]$ 是目标模板和细碎模板组合而 成 $\boldsymbol{x}^{j}=\left[\left(\boldsymbol{a}^{j}\right)^{\mathrm{T}},\left(\boldsymbol{e}^{j}\right)^{\mathrm{T}}\right]^{\mathrm{T}}$. 注意, 它要求所有 $\boldsymbol{b}_{i}^{j}$ 共享 相同的状态, 但不是所有的 $\boldsymbol{T}^{j}$, 因为每个精心预 选的 $\boldsymbol{T}^{j}$ 与其他 $\boldsymbol{T}^{j}$ 没有任何状态约束.

在实际应用中, $\boldsymbol{b}_{i}^{j}$ 经常含有噪声, 因此在这 种情况下, 等式约束可以放宽到不等式约束, 从而 为系数选择提供了更大的灵活性. 最后, 可把多模 态联合压缩表示归结为求解具有 $\ell_{2}$ 范数不等式约 束的 $\ell_{1}$ 最小化问题

$$
\min _{X} \sum_{j=1}^{J}\left\|\boldsymbol{x}^{j}\right\|_{1} \text { s.t. } \sum_{j=1}^{J}\left\|\boldsymbol{\Phi} \boldsymbol{A}^{j} \boldsymbol{x}^{j}-\boldsymbol{\Phi} \boldsymbol{b}_{i}^{j}\right\|_{2}^{2} \leqslant \varepsilon, \boldsymbol{X} \succeq \mathbf{0}
$$

其中, $\boldsymbol{X}=\left\{\boldsymbol{x}^{1}, \cdots, \boldsymbol{x}^{J}\right\} ; \varepsilon>0$ 是一个预先确定的噪 声级. 本文中, 由于不同的模态可能有利于不同的 稀疏重构系数，因此不同模态之间的联合稀疏性 约束是很有价值的, 但联合稀疏性可以增强系数 估计的鲁棒性.

\section{2 定制化 DALM 来求解式(10)}

本节将介绍使用 DALM 方法来有效地解决式 (10)的问题. 原始的 DALM 仅用于求解类似于式 (3)的单 $\ell_{1,2}$ 混合范数的极小化问题, 但在本文中, 需要解决多个 $\ell_{1,2}$ 混合范数组合的极小化问题. 此
外, 式(3)的对偶问题也有很大不同, 前者采用一 般等式约束, 后者采用 2-范数不等式约束. 因此, 必须特制 DALM 来求解式(10)中具有多重 $\ell_{1}$-范数 组合和 $\ell_{2}$-范数不等式约束的极小化问题.

要直接构建与 $x^{1}, \cdots, x^{J}$ 相关的对偶问题很困 难, 所以本文对式(10)做了一些变换. 如果使

$$
\boldsymbol{B} \doteq\left[\begin{array}{cccc}
\boldsymbol{B}^{1} & \mathbf{0} & \cdots & \mathbf{0} \\
\mathbf{0} & \boldsymbol{B}^{2} & \cdots & \mathbf{0} \\
\vdots & \vdots & \ddots & \vdots \\
\mathbf{0} & \mathbf{0} & \cdots & \boldsymbol{B}^{J}
\end{array}\right], \quad \boldsymbol{x} \doteq\left[\begin{array}{c}
\boldsymbol{x}^{1} \\
\boldsymbol{x}^{2} \\
\vdots \\
\boldsymbol{x}^{J}
\end{array}\right], \quad \boldsymbol{c}_{i} \doteq\left[\begin{array}{c}
\boldsymbol{c}_{i}^{1} \\
\boldsymbol{c}_{i}^{2} \\
\vdots \\
\boldsymbol{c}_{i}^{J}
\end{array}\right]
$$

其中, $\boldsymbol{B}^{j}=\boldsymbol{\Phi} \boldsymbol{A}^{j}, \boldsymbol{c}_{i}^{j}=\boldsymbol{\Phi} \boldsymbol{b}_{i}^{j} ; \boldsymbol{0}$ 是 $d \times(N+2 d)$ 的零 矩阵, 则式(10)可以等价地转换为有约束的基追踪 去噪 $\boldsymbol{B P}_{\delta}$ 问题

$$
\min _{\boldsymbol{x}}\|\boldsymbol{x}\|_{1} \quad \text { s.t. }\left\|\boldsymbol{B} \boldsymbol{x}-\boldsymbol{c}_{i}\right\|_{2} \leqslant \varepsilon, \boldsymbol{x} \succeq 0
$$

它的变换包括无约束的基追踪去噪 $\boldsymbol{B P}_{\delta}$ 问题

$$
\min _{\boldsymbol{x}}\|\boldsymbol{x}\|_{1} \quad \text { s.t. } \quad \frac{1}{2 \delta}\left\|\boldsymbol{B} \boldsymbol{x}-\boldsymbol{c}_{i}\right\|_{2}^{2} \leqslant \varepsilon, \boldsymbol{x} \succeq 0
$$

其中, $\delta>0$ 是预先设定的权重. 式(13)的对偶问题为

$$
\max _{\boldsymbol{y}} \boldsymbol{c}_{i}^{\mathrm{T}} \boldsymbol{y}-\frac{\delta}{2}\|\boldsymbol{y}\|_{2}^{2} \quad \text { s.t. } \quad \boldsymbol{B}^{\mathrm{T}} \boldsymbol{y} \in \boldsymbol{C}
$$

其增广拉格朗日问题是

$$
\begin{aligned}
& \min _{\boldsymbol{y}, \boldsymbol{z}}-\boldsymbol{c}_{i}^{\mathrm{T}} \boldsymbol{y}+\frac{\delta}{2}\|\boldsymbol{y}\|_{2}^{2}-\boldsymbol{x}^{\mathrm{T}}\left(\boldsymbol{z}-\boldsymbol{B}^{\mathrm{T}} \boldsymbol{y}\right)+\frac{\beta}{2}\left\|\boldsymbol{z}-\boldsymbol{B}^{\mathrm{T}} \boldsymbol{y}\right\|_{2}^{2}, \\
& \boldsymbol{z} \in \boldsymbol{C} .
\end{aligned}
$$

其中, $\boldsymbol{C}=\left\{\boldsymbol{\theta} \in \mathbb{R}^{J\left(N+2 d_{0}\right)}: \boldsymbol{\theta} \leqslant \mathbf{1}\right\} ; \boldsymbol{x}=\mathbb{R}^{J\left(N+2 d_{0}\right)}$ 为 拉格朗日乘子; $\boldsymbol{c}_{i} \in \mathbb{R}^{J d} ; \quad \boldsymbol{B} \in \mathbb{R}^{(J d) \times\left(J\left(N+2 d_{0}\right)\right)}$.

应用类似于第 1.2 节的变换策略, 得到从 $\left(\boldsymbol{x}_{k}, \boldsymbol{y}_{k}, \boldsymbol{z}_{k}\right)$ 到 $\left(\boldsymbol{x}_{k+1}, \boldsymbol{y}_{k+1}, \boldsymbol{z}_{k+1}\right)$ 的迭代算法为

$$
\left\{\begin{array}{l}
\boldsymbol{z}_{k+1}=\mathcal{P}_{\boldsymbol{C}}\left(\boldsymbol{B}^{\mathrm{T}} \boldsymbol{y}_{k}+\boldsymbol{x}_{k} / \beta\right) \\
\boldsymbol{y}_{k+1}=\left(\delta \boldsymbol{I}+\beta \boldsymbol{B} \boldsymbol{B}^{\mathrm{T}}\right)^{-1}\left(\beta \boldsymbol{B} \boldsymbol{z}_{k+1}-\left(\boldsymbol{B} \boldsymbol{x}_{k}-\boldsymbol{c}_{i}\right)\right) \\
\boldsymbol{x}_{k+1}=\boldsymbol{x}_{k}-\beta\left(\boldsymbol{z}_{k+1}-\boldsymbol{B}^{\mathrm{T}} \boldsymbol{y}_{k+1}\right)
\end{array}\right.
$$

其中, $\left(\delta \boldsymbol{I}+\beta \boldsymbol{B} \boldsymbol{B}^{\mathrm{T}}\right)$ 是可逆的. 特别是当 $\delta=0, J=1$ 时, 式(15)将退化为式(6) 式(8).

式(15)涉及许多矩阵向量乘法, 如 $\boldsymbol{B} \boldsymbol{x}, \boldsymbol{B} z$ 和 $\boldsymbol{B}^{\mathrm{T}} \boldsymbol{y}$ 等, 直接计算它们的成本较高, 但可以使用

$$
\boldsymbol{B} \boldsymbol{x}=\left[\begin{array}{c}
\boldsymbol{B}^{1} \boldsymbol{x}^{1} \\
\boldsymbol{B}^{2} \boldsymbol{x}^{2} \\
\vdots \\
\boldsymbol{B}^{J} \boldsymbol{x}^{J}
\end{array}\right], \boldsymbol{B} \boldsymbol{z}=\left[\begin{array}{c}
\boldsymbol{B}^{1} \boldsymbol{z}^{1} \\
\boldsymbol{B}^{2} \boldsymbol{z}^{2} \\
\vdots \\
\boldsymbol{B}^{J} \boldsymbol{z}^{J}
\end{array}\right], \boldsymbol{B}^{\mathrm{T}} \boldsymbol{y}=\left[\begin{array}{c}
\left(\boldsymbol{B}^{1}\right)^{\mathrm{T}} \boldsymbol{y}^{1} \\
\left(\boldsymbol{B}^{2}\right)^{\mathrm{T}} \boldsymbol{y}^{2} \\
\vdots \\
\left(\boldsymbol{B}^{J}\right)^{\mathrm{T}} \boldsymbol{y}^{J}
\end{array}\right]
$$

进行等价计算. 式(15)中矩阵求逆的计算量虽然很 大, 但是它只需计算一次, 并且在当前帧的跟踪中 保持不变. 此外, $\delta \boldsymbol{I}+\beta \boldsymbol{B} \boldsymbol{B}^{\mathrm{T}}=\operatorname{diag}\left(\delta \boldsymbol{I}^{1}+\beta \boldsymbol{B}^{1}\left(\boldsymbol{B}^{1}\right)^{\mathrm{T}}, \cdots\right.$, 
$\left.\delta \boldsymbol{I}^{J}+\beta \boldsymbol{B}^{J}\left(\boldsymbol{B}^{J}\right)^{\mathrm{T}}\right)$ 为对角方阵, 它的逆矩阵等于 $\operatorname{diag}\left(\left[\delta \boldsymbol{I}^{1}+\beta \boldsymbol{B}^{1}\left(\boldsymbol{B}^{1}\right)^{\mathrm{T}}\right]^{-1}, \cdots,\left[\delta \boldsymbol{I}^{J}+\beta \boldsymbol{B}^{J}\left(\boldsymbol{B}^{J}\right)^{\mathrm{T}}\right]^{-1}\right)$ ，通 过计算所有的 $\left[\delta \boldsymbol{I}^{j}+\beta \boldsymbol{B}^{j}\left(\boldsymbol{B}^{j}\right)^{\mathrm{T}}\right]^{-1}$, 就可以进一步 减小求逆的计算量, 其中 $\boldsymbol{I}^{j} \in \mathbb{R}^{d \times d}$ 为单位矩阵.

对状态 $S_{i}$ 的每个观测 $\boldsymbol{b}_{i}=\left[\left(\boldsymbol{b}_{i}^{1}\right)^{\mathrm{T}}, \cdots,\left(\boldsymbol{b}_{i}^{J}\right)^{\mathrm{T}}\right]^{\mathrm{T}}$, 本文采用特定的 DALM 方法, 通过求解问题式 (13) 来恢复目标系数向量 $\boldsymbol{a}_{i}=\left[\left(\boldsymbol{a}_{i}^{1}\right)^{\mathrm{T}}, \cdots,\left(\boldsymbol{a}_{i}^{J}\right)^{\mathrm{T}}\right]^{\mathrm{T}}$. 其残差为

$$
r_{i}=\sqrt{\sum_{j=1}^{J}\left\|\boldsymbol{\Phi} \boldsymbol{T}^{j} \boldsymbol{a}_{i}^{j}-\boldsymbol{\Phi} \boldsymbol{b}_{i}^{j}\right\|_{2}^{2}}
$$

观测似然估计通过 $l_{i}=\exp \left(-r, r_{i}\right)$ 计算. 最后, 在帧 $t$ 中以均方误差方法来代替文献[2]中使用的 最大似然估计，来更新目标状态，即

$$
\boldsymbol{s}_{t}=\sum_{i=1}^{M} l_{i} \cdot \mathbf{s}_{i} / \sum_{i=1}^{M} l_{i}
$$

多模态联合压缩跟踪 (multimodal joint compressive tracking, MJCT)过程如算法 1, 其中的模 板更新算法将在第 2.3 节详细介绍. 模板更新和残 差计算的计算成本相对较低, 其计算量主要由矩 阵求逆和矩阵向量乘法组成, 因此, 算法 1 的复杂 度近似等于 $O\left(M \cdot m \cdot J \cdot d \cdot\left(N+2 d_{0}\right)\right), m$ 是 DALM 过程的平均迭代次数.

\section{算法 1. MJCT.}

输入. 当前帧 $\left\{F_{t}^{j}\right\}_{j=1}^{J}$ 、采样状态 $\left\{\boldsymbol{s}_{i}\right\}_{i=1}^{M}$ 、模板 集 $\left\{\boldsymbol{A}^{j}=\left[\boldsymbol{T}^{j}, \boldsymbol{E}^{j}\right] \in \mathbb{R}^{d_{0} \times\left(N+2 d_{0}\right)}\right\}_{j=1}^{J}$ 、惩罚系数 $\beta>0$ 、 权值 $\delta>0$ 、常量 $\kappa>0$ 、降维矩阵 $\boldsymbol{\Phi} \in \mathbb{R}^{d \times d_{0}}$.

输出. 新的目标状态 $\boldsymbol{s}_{t}$ 和目标模板集 $\left\{\boldsymbol{T}^{j}\right\}_{j=1}^{J}$.

Step1. $\boldsymbol{B}^{j} \leftarrow \boldsymbol{\Phi} \boldsymbol{A}^{j}$ ，并归一化所有 $\boldsymbol{B}^{j}$ 的每一列.

Step2. for $i=1$ to $M$ :

Step2.1. 对所有模态 $j$, 获得其状态 $s_{i}$ 的归一化 系数 $\boldsymbol{c}_{i}^{j} \leftarrow \boldsymbol{\Phi} \boldsymbol{b}_{i}^{j}$;

Step2.2. 对所有模态 $j$, 初始化 $\boldsymbol{x}_{1} \in \mathbb{R}^{N+2 d_{0}}$ 为 $\mathbf{0}$, $\boldsymbol{y}_{1} \in \mathbb{R}^{J \times d}$ 为 $\mathbf{0}$;

Step2.3. 根据式(11)构造 $\boldsymbol{B}, \boldsymbol{c}_{i}$ 和 $\boldsymbol{x}$, 执行 $x_{1} \leftarrow x$

Step2.4. while 未收敛 $(k=1,2, \cdots)$ :

Step2.4.1. $\boldsymbol{z}_{k+1}=\mathcal{P}_{C}\left(\boldsymbol{B}^{\mathrm{T}} \boldsymbol{y}_{k+1}+\boldsymbol{x}_{k} / \beta\right)$;

Step2.4.2. $\quad \boldsymbol{y}_{k+1}=\left(\delta I+\beta \boldsymbol{B B}^{\mathrm{T}}\right)^{-1}\left(\beta \boldsymbol{B} \boldsymbol{z}_{k+1}-\left(\boldsymbol{B}^{\mathrm{T}} \boldsymbol{x}_{k}-\right.\right.$ $\left.\left.c_{i}\right)\right)$;

Step2.4.3. $\boldsymbol{x}_{k+1}=\boldsymbol{x}_{k+1}-\beta\left(z_{k+1}-\boldsymbol{B}^{\mathrm{T}} z_{k+1}\right)$;

Step2.5. 对所有模态 $j$, 执行 $\boldsymbol{a}_{i}^{j} \leftarrow \boldsymbol{x}_{k}[(j-1)(N+$ $\left.\left.2 d_{0}\right)+1:(j-1)\left(N+2 d_{0}\right)+N\right]$;

Step2.6. 通过式(16)计算残差 $r_{i}$, 并计算 $l_{i}=$

$\exp \left(-\kappa \cdot r_{i}\right)$

Step3. 按式(17)计算目标状态 $\boldsymbol{s}_{t}$, 再获得目标 $\boldsymbol{b}_{t}$.

Step4. 归一化 $\boldsymbol{\Phi} \boldsymbol{b}_{t}$, 并根据式(13)重新计算 $\boldsymbol{s}_{t}$.

Step5. 通过协同更新方法更新模板集 $\left\{\boldsymbol{T}^{j}\right\}_{j=1}^{J}$.

\section{3 模板更新}

在实际的跟踪中, 应及时更新目标模板集, 以 便跟踪器能够捕捉由于光照或姿态变化等引起的 目标外观变化. 本文提出一种协作更新策略来解 决这个问题。

每个模板的重要性应该根据稀疏系数的分布 来判断, 故可以借鉴稀疏密集指数 (sparsity concentration index, $\mathrm{SCI})^{[23]}$ 来进行模板更新. 为了说 明这一做法的合理性, 选择跟踪过程中的一些中 间结果进行观察. 如图 2 所示, 其中蓝色的针状线 (1 190) 代表可见光图像特征, 红色的针状线 (191 380)代表红外图像特征; 图 2a 所示为所有候 选者能被其目标模板完美逼近的情况, 图 $2 \mathrm{~b}$ 所示 为仅有一个候选者能被其目标模板完美逼近的情 况, 图 2c 所示为所有候选者均未被其目标模板逼 近的情况. 不难发现, 与图 2a 和图 $2 \mathrm{~b}$ 中有效采样 状态的系数相比, 图 2c 中与无效采样状态相关联 的系数并不集中在任何一个模态上, 而是广泛分 布于整个模板集. 通过上面的观察不难发现, 稀疏 系数的分布包含关于采样状态有效性的重要信息: 一个有效的采样状态应该具有稀疏表示, 其非零 项主要集中在至少一个模态上; 而无效采样状态 的稀疏系数广泛分布于整个模板集. 为了量化这 一观察，本文将 SCI 定义修改为

$$
\left\{\begin{array}{l}
\operatorname{SCI}\left(\boldsymbol{x}_{t}^{j}\right)=\left(J \cdot\left\|\boldsymbol{x}_{t}^{j}\right\|_{1} /\left\|\boldsymbol{x}_{t}\right\|_{1}-1\right) /(J-1) \in[0,1] \\
\operatorname{SCI}\left(\boldsymbol{a}_{t}^{j}\right)=\left(N \cdot\left\|\boldsymbol{a}_{t}^{j}\right\|_{1} /\left\|\boldsymbol{x}_{t}^{j}\right\|_{1}-1\right) /(N-1) \in[0,1]
\end{array}\right.
$$

其中, $\operatorname{SCI}\left(x_{t}^{j}\right)$ 指所有模态间稀疏系数的密集度; 而 $\operatorname{SCI}\left(a_{t}^{j}\right)$ 是指在一个模态上的稀疏系数密集度. 对于 $\boldsymbol{x}_{t}$, 如果 $\max _{j} \operatorname{SCI}\left(\boldsymbol{x}_{t}^{j}\right)=1$, 则所有模态候选中 只有一个近似模板; 如果 $\max _{j} \operatorname{SCI}\left(x_{t}^{j}\right)=0$, 则稀疏 系数均匀地分布在所有模态上, 这意味着所有模 态候选中没有一个能其近似模板.

由上述分析可知, 一个模态是否需要更新是 由它的系数集中度与其他模态的关系所决定的. 因此，可将该模板更新方法命名为协同更新方法, 包括模态选择、模板选择和模板替换. 如果是 

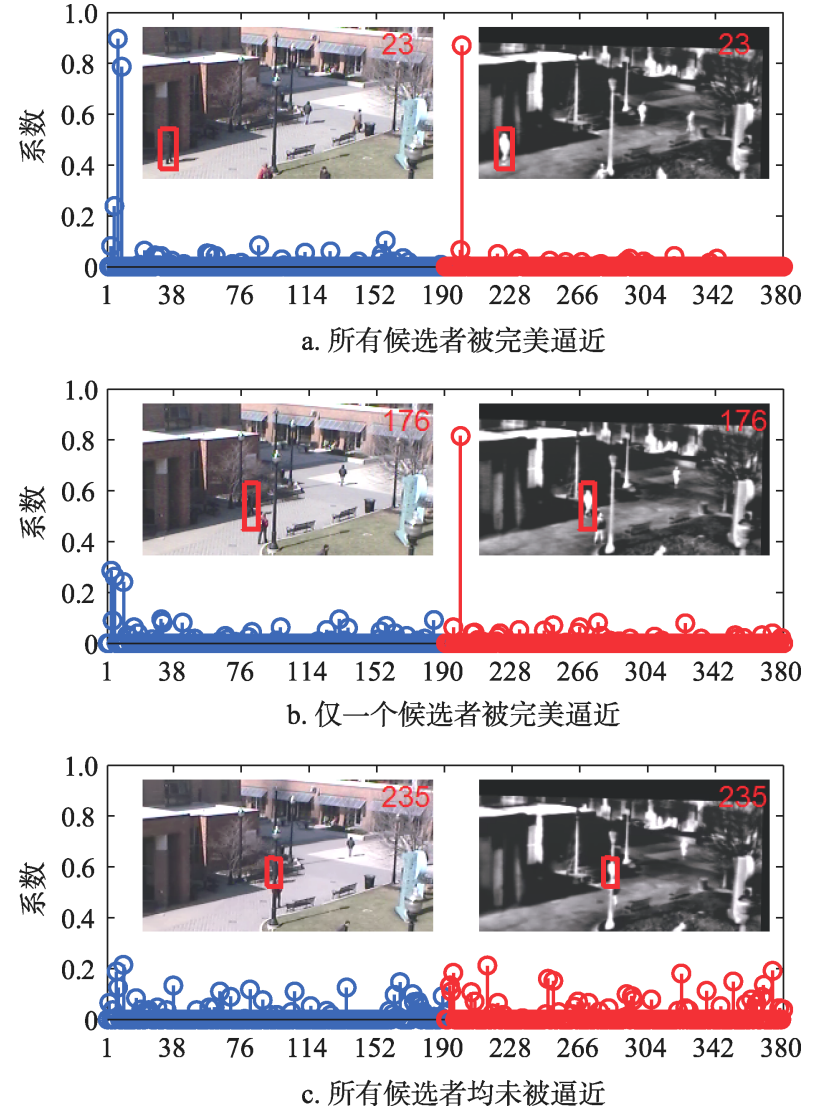

图 2 有效采样状态和无效采样状态对应的 稀疏系数分布图

$\operatorname{SCI}\left(\boldsymbol{x}_{t}^{j}\right) \leqslant T_{1}$ 和 $\operatorname{SCI}\left(\boldsymbol{a}_{t}^{j}\right) \leqslant T_{2}$, 那么可把第 $j$ 个模态 作为需要更新的候选模板, 其中 $T_{1}, T_{2} \in(0,1)$ 是 2 个预先设置的阈值. 对于每个候选模板, 如果其跟 踪结果与当前模板集不相似, 则它将用相似性代 替最不重要的模板. 算法 2 总结了模板更新方案.

算法 2. 协同更新方法.

输入. 第 $t$ 帧的跟踪结果 $\boldsymbol{b}_{t}=\left[\left(\boldsymbol{b}_{t}^{1}\right)^{\mathrm{T}}, \cdots,\left(\boldsymbol{b}_{t}^{J}\right)^{\mathrm{T}}\right]^{\mathrm{T}}$ 、 稀疏系数 $\boldsymbol{x}_{t}=\left[\left(\boldsymbol{x}_{t}^{1}\right)^{\mathrm{T}}, \cdots,\left(\boldsymbol{x}_{t}^{J}\right)^{\mathrm{T}}\right]^{\mathrm{T}}$ 、阈值 $\tau_{1}$ 和 $\tau_{2}$.

输出. 更新后的模板集.

Step1. for $j=1$ to $J$ :

Step1.1. 通过式(18)计算 $\operatorname{SCI}\left(\boldsymbol{x}_{i}^{j}\right)$ 和 $\operatorname{SCI}\left(\boldsymbol{a}_{t}^{j}\right)$;

Step1.2. if $\left(\operatorname{SCI}\left(\boldsymbol{x}_{t}^{j}\right)<\tau_{1}\right.$ 且 $\left.\operatorname{SCI}\left(\boldsymbol{a}_{t}^{j}\right)<\tau_{2}\right)$ :

Step1.2.1. 执行 $\hat{n} \leftarrow \underset{1 \leqslant n \leqslant N}{\arg \min } \operatorname{sim}\left(\boldsymbol{t}_{n}^{j}, \boldsymbol{b}_{t}^{j}\right)$;

Step1.2.2. 执行 $\boldsymbol{t}_{\hat{n}}^{j} \leftarrow \boldsymbol{b}_{t}^{j} ; / /$ 代替一个旧模板.

说明: 给定稀疏投影矩阵 $\boldsymbol{\Phi} \in \mathbb{R}^{d \times d_{0}}$ ，对于 $d$ 有 2 个约束规则, 第 1 个是服从 Johnson-Lindenstrauss

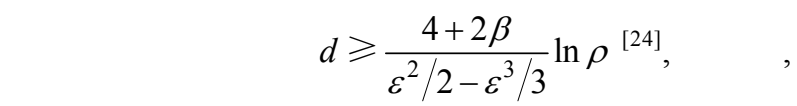
$\beta>0,0<\varepsilon<1, \rho \in \mathbb{R}^{d_{0}}$. 在实践中, 这一约束 远比在图像和文本数据上取得的效果要好. 在本 文实验中 $\rho=190, \varepsilon=0.2, \beta=1$, 则下界 $d \approx 1800$. 另一个由压缩传感中的限制等距性质导出的约束 $d \geqslant \kappa \beta \ln \left(d_{0} / \beta\right)$, 其中, $\kappa, \beta$ 为常数. 它比理论边 界要紧得多. 当 $d_{0}=180, \kappa=1, \beta=10$ 时, $d \geqslant 12$. 实验表明, $d=50$ 时足以产生良好的结果.

\section{3 实验与分析}

本文使用了 9 个已配准好的红外-可见序列来 测评所提出的方法, 其中 1 个为自制, 其余 8 个选 自公共数据集 $\mathrm{DCU}^{[18]}, \mathrm{OTCBVS}^{\circledR}$ 和 $\mathrm{BEPMDS}^{\circledR}$, 还从公共数据集 $\mathrm{OTB} 50^{\circledR}$ 和 VOT-TIR ${ }^{\oplus}$ 中分别选取 了 4 个可见序列和 4 个红外序列(表 1 列出了这些 序列的详情). 采用一次通过的评估方法将本文方 法与目前最先进的跟踪器进行了比较, 这些跟踪 器包括 $\mathrm{JSRT}^{[17]}, \mathrm{FRD}^{[14]}, \mathrm{L}^{\left[T^{[2]}\right.}, \mathrm{KCF}^{[6]}$ 和 $\mathrm{L} 1 \mathrm{APG}^{[3]}$; 其中前 2 种是红外-可见光融合跟踪器, 后 3 种是

表 1 实验中被测图像序列详情

\begin{tabular}{lllll}
\hline 图像序列 & 名称 & 尺寸 & 来源 & \multicolumn{1}{c}{ 属性 } \\
\hline & nigher & $640 \times 480$ & DCU & 无光的夜晚 \\
& cluter & $640 \times 480$ & DCU & 背景拥簇 \\
& inrota & $640 \times 480$ & DCU & 平面内旋转 \\
& fullocc & $288 \times 240$ & BEPMDS & 全遮挡 \\
红外-可见光 & parocc & $320 \times 240$ & OTCBVS & 部分遮挡 \\
& croser & $320 \times 240$ & OTCBVS & 目标交叉 \\
& shader & $320 \times 240$ & OTCBVS & 步行在阴影中 \\
& jumper & $480 \times 360$ & 自制序列 & 形变 \\
& outrota & $640 \times 480$ & DCU & 平面外旋转 \\
\hline \multirow{4}{*}{191 帧 } & car4 & $360 \times 240$ & OTB50 & 伸缩和阴影 \\
可见光 & coupon & $320 \times 240$ & OTB50 & 背景拥簇 \\
& faceocc1 & $352 \times 288$ & OTB50 & 遮挡 \\
& jumper & $352 \times 288$ & OTB50 & 快速运动模糊 \\
\hline \multirow{3}{*}{1948 帧 } & rhino & $320 \times 256$ & VOT-TIR & 运动改变 \\
红外 & horse & $324 \times 256$ & VOT-TIR & 形变 \\
& street & $640 \times 480$ & VOT-TIR & 交汇 \\
& trees1 & $640 \times 480$ & VOT-TIR & 遮挡 \\
\hline
\end{tabular}

(1) http://vcipl-okstate.org/pbvs/bench/

(2) http://www.cis.rit.edu/pelz/scanpaths/data/bristol-eden.htm

(3) http://cvlab.hanyang.ac.kr/tracker_benchmark/index.html

(4) http://votchallenge.net 
单源跟踪器. 本文方法是用 Matlab $+M E X$ 实现的, 并设置目标模板数 $N=10$, 模板尺寸 $d_{0}=180$, 粒 子数 $M=300, \tau_{1}=\tau_{2}=0.3$. 所有的跟踪结果是在 英特尔双核 $2.6 \mathrm{GHz}$ CPU 和 $8 \mathrm{~GB}$ RAM 上运行得 到的, 并使用相同的初始位置以示公平性.

使用文献[19]中建议的 5 个评价标准, 它们分 别是中心位置误差 $\varrho=\sqrt{\left(x_{\mathrm{G}}-x_{\mathrm{T}}\right)^{2}+\left(y_{\mathrm{G}}-y_{\mathrm{T}}\right)^{2}}$, 精 度 $p=\frac{1}{m} \sum_{i=1}^{m} h\left(\xi_{1}-\varrho_{i}\right)$, 边界框重叠率 $\varepsilon=\frac{\left|R_{\mathrm{G}} \cap R_{\mathrm{T}}\right|}{\left|R_{\mathrm{G}} \cup R_{\mathrm{T}}\right|}$, 成功率 $s=\frac{1}{m} \sum_{i=1}^{m} h\left(\varepsilon_{i}-\xi_{2}\right)$ 和帧率 $f=\frac{m}{t}$. 其中, $|\cdot|$ 表
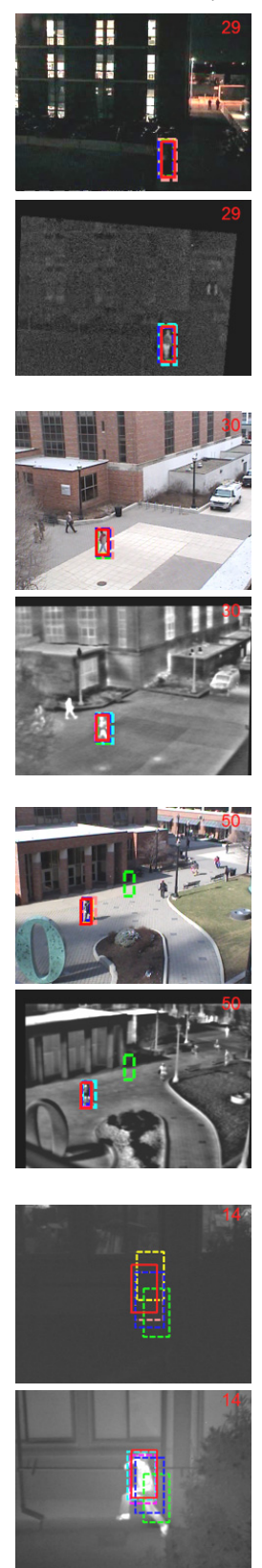

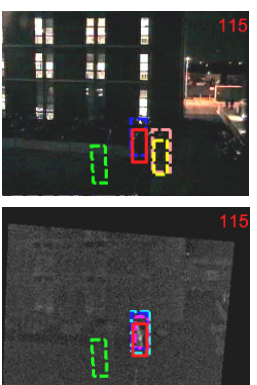

a. nigher
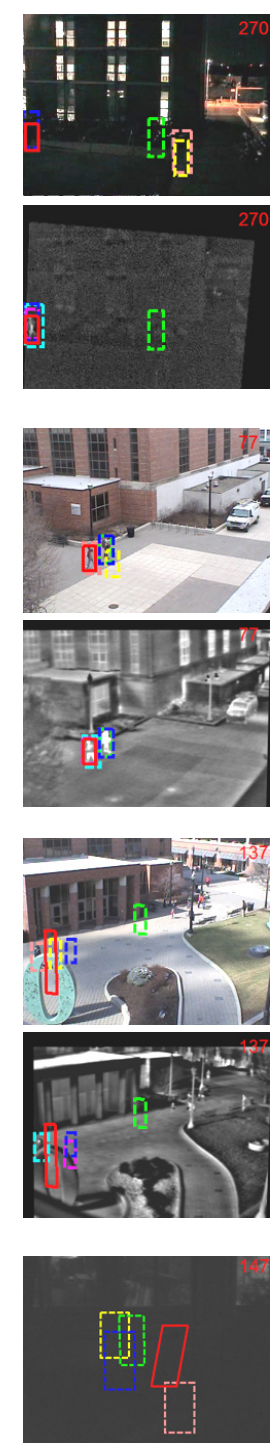

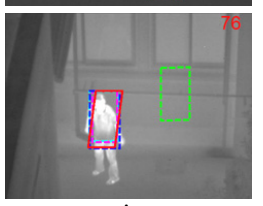

g. jumper

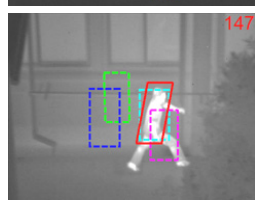

$-\operatorname{MJCT}$ (本文); - - - JSRT ${ }^{[17]}$
示像素数; $h(x)$ 是阶跃函数 (如果 $x \geqslant 0$, 那么 $h(x)=1$, 否则 $h(x)=0) ; \xi_{1}$ 和 $\xi_{2}$ 是 2 个國值, $\left(x_{\mathrm{T}}, y_{\mathrm{T}}, R_{\mathrm{T}}\right)$ 表示跟踪器提供的中心和边界框, $\left(x_{\mathrm{G}}, y_{\mathrm{G}}, R_{\mathrm{G}}\right)$ 表示对被跟踪对象进行人工标注生成 的地面事实; $m$ 是序列的长度; $t$ 是跟踪的总时间. 当边界框与地面事实重叠时, 重叠积分值为 1 , 此 时获得最佳跟踪结果.

\section{1 在红外-可见光序列上测评}

\section{1 .1 仅用灰度特征}

首先，选择原始像素值(即灰度)作为特征对红 外-可见光序列队进行测评. 图 3 显示了对表 1 中
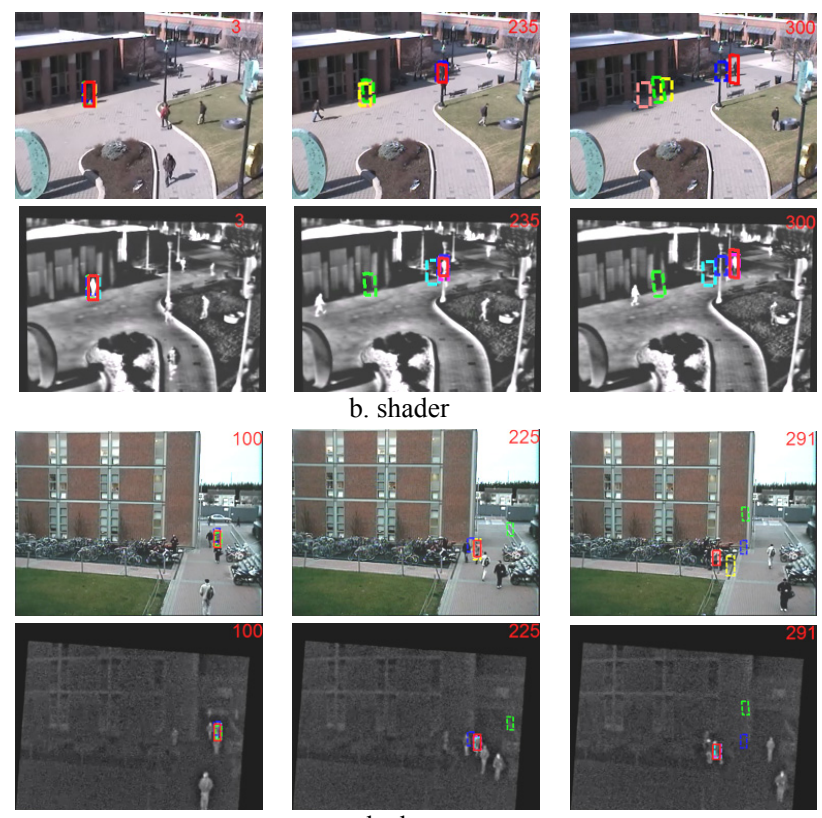

b. shader
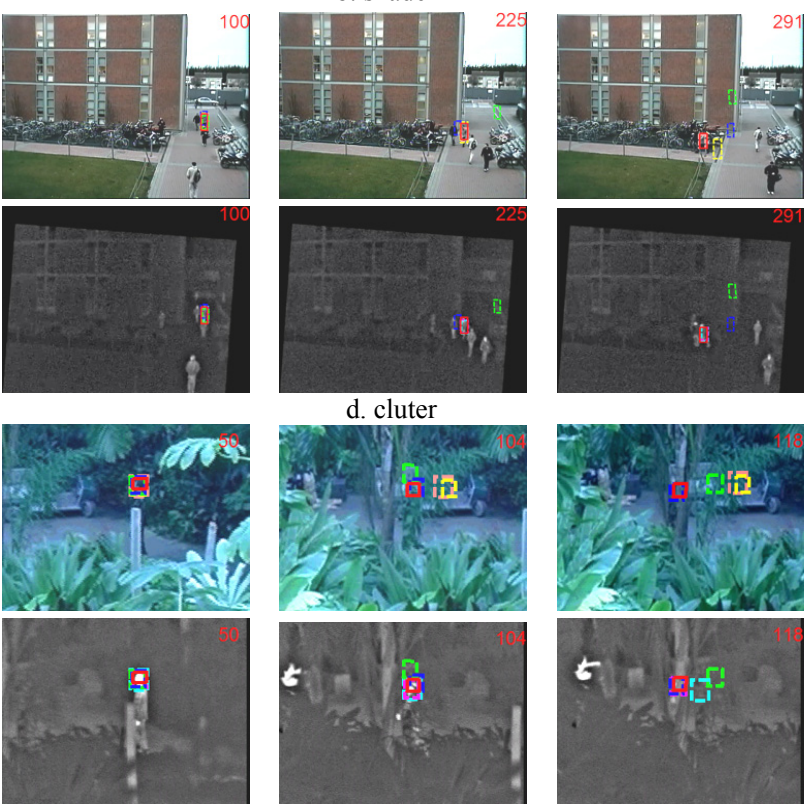

f. fullocc
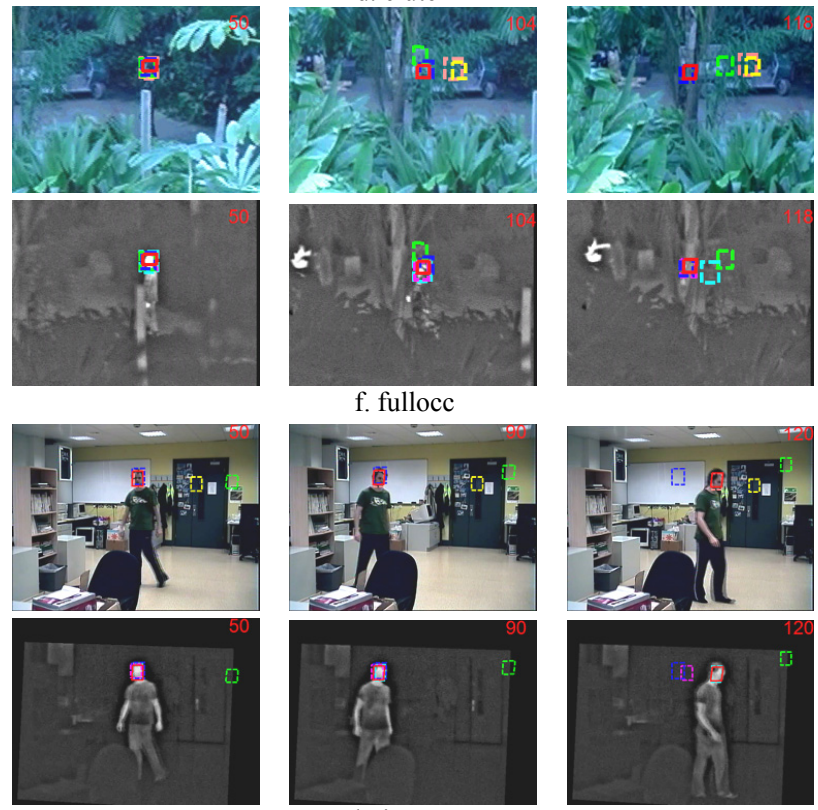

h. inrota

图 3 前 8 个红外-可见光序列中部分关键帧的跟踪结果截图 
前 8 个序列跟踪所得结果的一些屏幕截图, 在每个 子图中, 上行是可见光图像, 下行是红外图像. L1T-IR 和 KCF-IR 代表分别使用 L1T 和 KCF 跟踪 红外目标, L1T-VS 和 KCF-VS 则分别代表跟踪可 见光目标. 图 4 所示为逐帧定位误差和重叠率. 由 于 JSRT 只使用单一目标模板, 导致了稀疏表示多 样性的损失, 所以其跟踪效果在所有序列都很差.

夜晚和阴影. 如图 $3 \mathrm{a}$ 和图 $3 \mathrm{~g}$ 所示, 在夜晚无 光的情况下, 可见光的图像目标通常是模糊的, 因 此只使用可见光的跟踪器, 如 L1T-VS 和 KCF-VS,
往往无法锁定目标. 然而, 通过红外摄像机, 如 L1T-IR, KCF-IR, MJCT 和 FRD 等跟踪器, 就能很好 地锁定目标, 因为红外传感器不受光照影响. 在图 $3 b$ 中, 黑衣人正在进入一个阴影区域, 在可见光图 像上, 他与背景间的对比度很低, 但在红外图像上 就不是这样; 因此 MJCT, FRD, KCF-IR 和 L1T-IR 能 够很好地锁定目标, 而 L1T-VS 和 KCF-VS 则不能. L1T-IR 和 MJCT 都成功地克服了灯柱的遮挡(如图 $3 b$ 中的第 235 帧和第 300 帧), 但 FRD 和 KCF 均失败 了, 这主要是由于 MJCT 和 L1T-IR 使用了细碎模板.
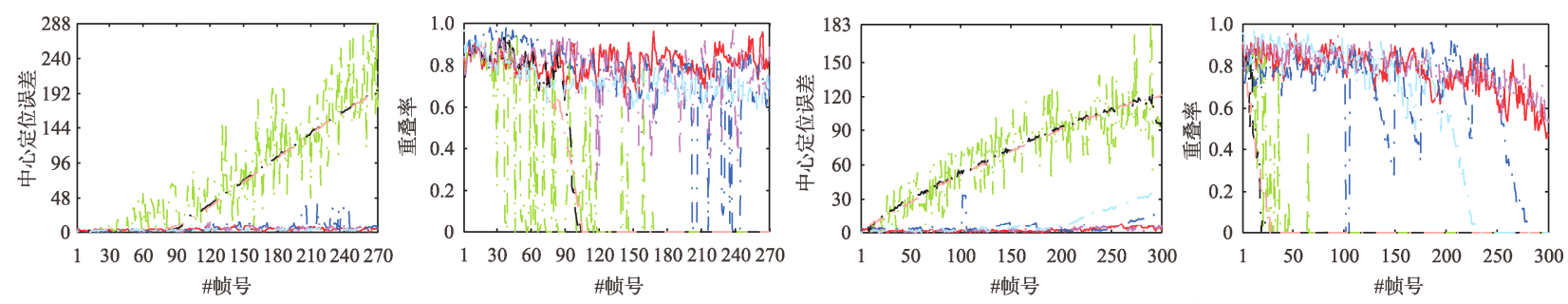

a. nigher
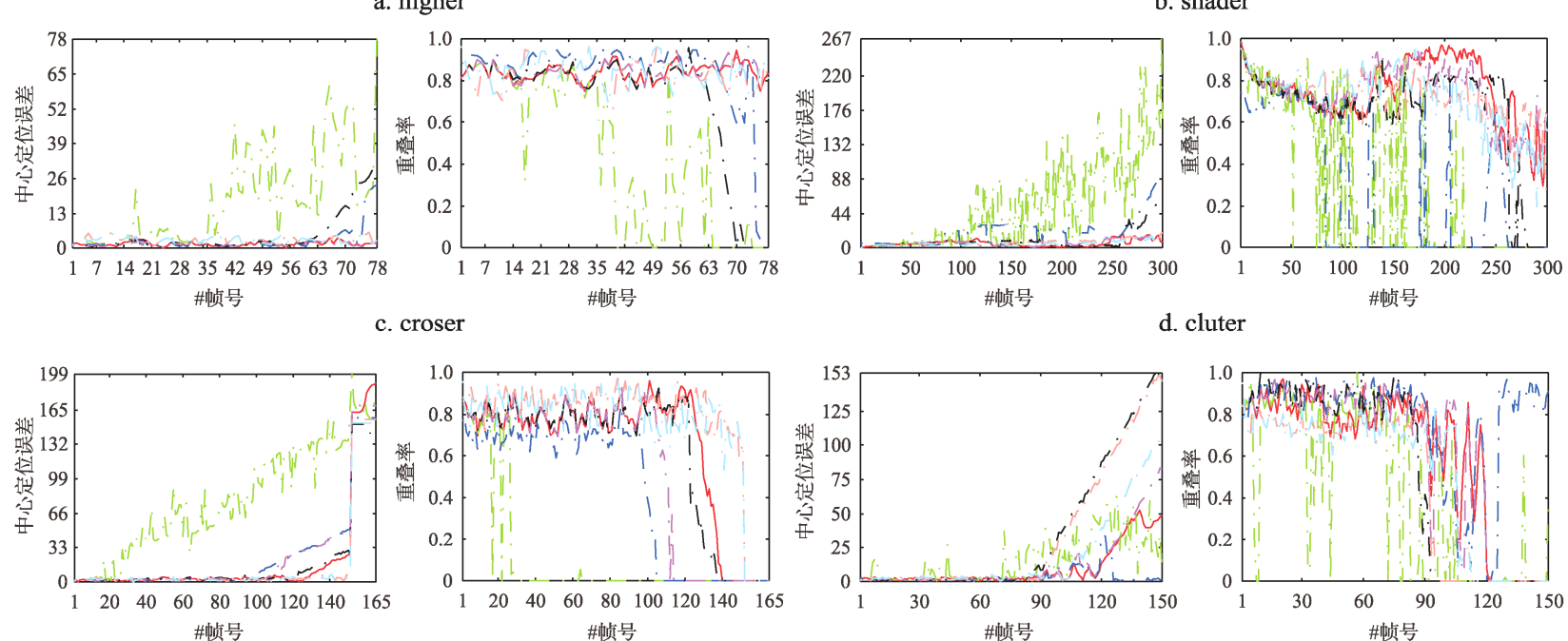

e. parocc
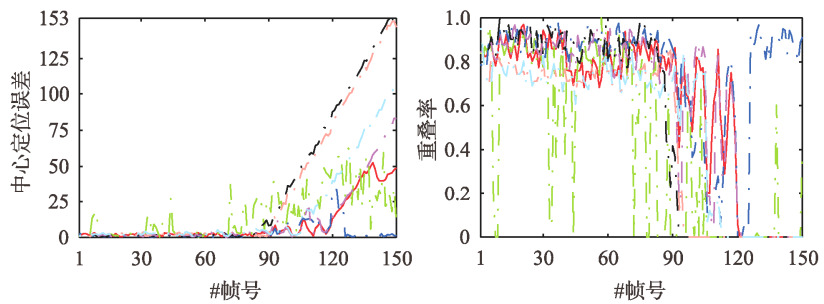

f. fullocc
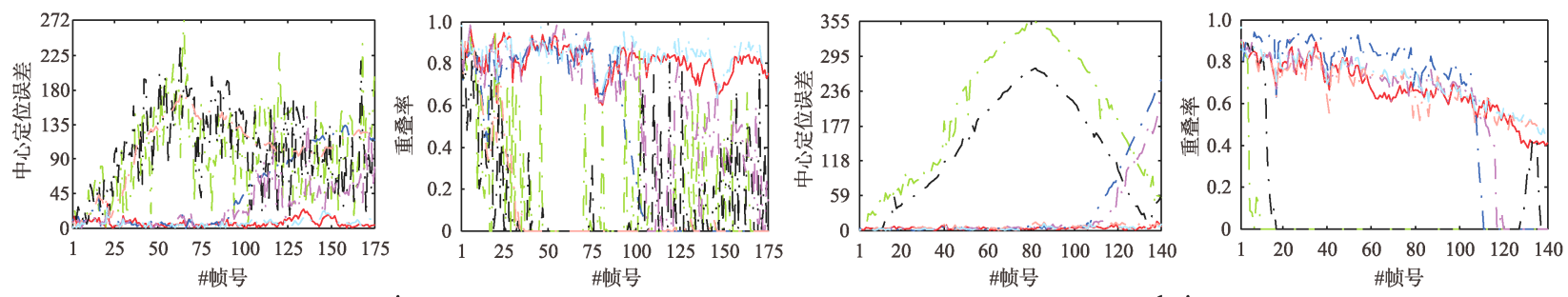

h. inrota

- MJCT(本文); - - - JSRT ${ }^{[17]}$; - - - FRD ${ }^{[14]}$; - - - L1T-VS ${ }^{[2]}$; - - -L1T-IR ${ }^{[2]}$; - - - KCF-VS ${ }^{[6]}$;

图 4 各跟踪方法在前 8 个红外-可见光序列上的中心定位误差和重叠率

交叉和混乱. 当目标处于热交汇或混乱的场 景时, 跟踪器很容易被干扰物所吸引. 参见图 $3 \mathrm{c}$ 中的第 77 帧, 当 2 个人处于热交汇时, FRD 和 JSRT 被正确的人所吸引, FRD 和 L1T-VS 被杂乱的 背景所吸引(如图 3d 中的第 225 帧和第 291 帧). 干
扰物的外观与目标相似, 可以很好地匹配目标, 所 以很难只使用单一光源的匹配得分来区分这种干 扰. 然而, 由于 MJCT 使用红外和可见光特征的联 合压缩表示, 使得其可以始终跟踪到目标. 由于使 用了判别模型, KCF 也取得了较好的效果. 
局部或全遮挡. 遮挡是目标跟踪中最普遍而又 关键的问题. 对于局部遮挡, KCF 表现最好, MJCT 和 L1T-IR 使用一般的模板来处理也可以克服它(如 图 3b 的第 235 帧, 图 3e 中的第 103 帧, 图 3f 中的 第 104 帧). 然而, 其他跟踪器都会偏离目标. 如图 4f 所示, 对于完全遮挡, 所有跟踪器都跟丢了.

变形和旋转. 在 jumper 序列中, 人在上下跳 跃时会产生很大的形变. 图 $3 \mathrm{~g}$ 给出了序列的跟踪 结果. 相比于其他跟踪器, MJCT 更容易适应形变, 这主要是由于 2 个方面: (1) 利用仿射运动模型; (2) 对红外和可见光特征进行了联合压缩表示. 如 图 4h 所示, 男子的头发生了大幅度旋转, 因此从 第 115 帧开始, FRD 和 L1-TIR 的跟踪框飘离了目 标. 而本文方法在整个序列中表现良好, 这是因为
仿射运动模型和协同模板更新策略可以处理复杂 运动中目标外观的变化.

$\mathrm{L} 1 \mathrm{~T}$ 和 $\mathrm{KCF}$ 是一种单模态跟踪器, 为便于与 其他融合跟踪器进行比较, 将可见光和红外的跟 踪结果综合成如下: (1) 跟踪器的速度等于 2 个序 列的长度除以处理时间; (2) 目标状态等于跟踪器 提供的红外和可见光目标状态的重加权平均, 其 中权重是由它们与真实情况的相似性所决定的. 表 2 给出了表 1 中所有跟踪器的精度(在 $\xi_{1}=20$ 像 素处)、成功率 ( $\xi_{2}=0.5$ 重叠比)和每秒对所有跟踪 器的处理帧. 可以看出, 与其他跟踪器相比, MJCT 获得的精度和成功率最高, KCF 是最快的. 本文跟踪器采用压缩传感减少 $\ell_{1}$ 问题的维数, 所 以排在第 3 .

表 2 所有跟踪方法在表 1 中前 8 个图像序列上的跟踪精度、成功率和帧率

\begin{tabular}{|c|c|c|c|c|c|c|c|c|c|c|c|c|c|c|c|}
\hline \multirow{2}{*}{ 序列名 } & \multicolumn{3}{|c|}{ MJCT(本文) } & \multicolumn{3}{|c|}{ JSRT $^{[17]}$} & \multicolumn{3}{|c|}{$\mathrm{FRD}^{[14]}$} & \multicolumn{3}{|c|}{$\mathrm{L} 1 \mathrm{~T}^{[2]}$} & \multicolumn{3}{|c|}{$\mathrm{KCF}^{[6]}$} \\
\hline & 精度 & 成功率 & 帧率 & 精度 & 成功率 & 帧率 & 精度 & 成功率 & 帧率 & 精度 & 成功率 & 帧率 & 精度 & 成功率 & 帧率 \\
\hline nigher & 1.00 & 1.00 & 3.45 & 0.26 & 0.21 & 16.7 & 0.93 & 0.93 & 3.03 & 0.43 & 0.36 & 1.69 & 0.69 & 0.37 & 293 \\
\hline shader & 1.00 & 0.98 & 3.57 & 0.15 & 0.07 & 16.7 & 0.98 & 0.77 & 3.03 & 0.23 & 0.06 & 1.12 & 0.46 & 0.09 & 568 \\
\hline croser & 1.00 & 1.00 & 3.57 & 0.63 & 0.51 & 16.7 & 0.96 & 0.95 & 2.94 & 1.00 & 0.90 & 1.18 & 1.00 & 1.00 & 526 \\
\hline cluter & 1.00 & 0.93 & 3.13 & 0.41 & 0.38 & 11.1 & 0.49 & 0.36 & 2.94 & 0.91 & 0.87 & 0.76 & 1.00 & 0.95 & 720 \\
\hline parocc & 0.85 & 0.78 & 3.13 & 0.15 & 0.15 & 16.7 & 0.68 & 0.59 & 3.03 & 0.76 & 0.68 & 1.61 & 0.92 & 0.92 & 566 \\
\hline fullocc & 0.82 & 0.73 & 3.70 & 0.62 & 0.51 & 14.3 & 0.97 & 0.85 & 2.94 & 0.67 & 0.61 & 1.29 & 0.69 & 0.61 & 695 \\
\hline jumper & 0.97 & 1.00 & 4.17 & 0.10 & 0.19 & 14.3 & 0.48 & 0.51 & 3.03 & 0.20 & 0.31 & 1.39 & 0.53 & 0.18 & 143 \\
\hline inrota & 1.00 & 0.88 & 3.13 & 0.03 & 0.03 & 11.1 & 0.76 & 0.76 & 2.78 & 0.16 & 0.11 & 1.20 & 1.00 & 0.96 & 322 \\
\hline mean & 0.96 & 0.91 & 3.48 & 0.29 & 0.26 & 14.7 & 0.78 & 0.72 & 2.97 & 0.55 & 0.49 & 1.28 & 0.79 & 0.64 & 479 \\
\hline
\end{tabular}

\section{1 .2 综合灰度、LBP 和 HOG 特征}

除了组合不同的光谱源原始灰度特征(例如第 3.1 .1 节所示)外, 本文的跟踪框架还可以接受来自 不同光谱图像的 LBP 和方向梯度直方图(histogram of oriented gradient, HOG)等中层特征的融合. 本 节将介绍 4 个特征跟踪系统，其特征包括可见灰 度、红外灰度、可见 LBP 和红外 HOG. LBP 技术 是描述图像纹理特征非常有效的方法, 它具有计 算速度快、旋转不变性强等优点, 广泛应用于纹理 分析、图像检索和目标跟踪等方面域. 在 LBP 中, 每个像素都被赋予一个纹理值, 该值可以与像素 的灰度值相结合来表示目标. LBP 算子通过用中心 值对图像的邻域进行阈值化, 并将结果看做是二 进制数, 从而对图像中的像素进行标记. LBP 算子 的 8-邻域版本被定义为

$$
\operatorname{LBP}(\boldsymbol{x})=\sum_{i=0}^{7} h\left(g_{i}-g_{\mathrm{c}}\right) 2^{i} .
$$

其中, $h(x)$ 是阶跃函数; $g_{\mathrm{c}}$ 对应于 8 -邻域内中心像 素 $x$ 的灰度值; $g_{i}$ 对应于邻域中第 $i$ 个像素灰度值. HOG 技术 ${ }^{[25]}$ 用于统计图像局部区域的梯度方 向出现频次, 可以很有效地描述物体形状特征. HOG 对几何和光度变换不敏感, 因此在目标检测 中得到了广泛的应用. HOG 主要提取步骤如下:

Step1. 在水平和垂直方向分别为 $[-1,0,1]$ 和 $[1,0,-1]^{\mathrm{T}}$ 的一维中点导数掩模计算每个像素的梯度.

Step2. 将图像窗口划分为 $6 \times 6$ 像素的细胞单元, 并 通过单元内每个像素向方向直方图投梯度加权票的方式 来计算该单元的局部方向梯度直方图.

Step3. 将每 $3 \times 3$ 个单元组合在一起, 形成一个更大 的空间连接块, 并归一化块内的所有单元.

Step4. 最后的 HOG 描述子则是由所有块中的每个 单元的归一化直方图的各分量连接而成的向量. 详情请 参阅文献[25].

本文实验中，使用每个细胞单元 9 个通道的直 方图就能获得很好的跟踪精度. 为了使 HOG 特征 
能像普通图像一样的使用, 本文采用 HOG 特征可 视化方法 ${ }^{[26]}$ 获得其二维图像.

通常, 可见光图像包含更多的纹理特征, 而形 状特征在红外图像中更明显, 因此可从可见光图 像中提取 LBP 特征, 从红外图像中提取 HOG 特征. 图 5 展示了 4 特征融合系统的在 outrota 序列上的
跟踪结果, 其中图 $5 \mathrm{a}$ 中的底部图像是其顶部可见 光图像的 LBP 图像, 图 $5 b$ 中的底部图像是其顶部 红外图像的 HOG 图像, 图 5c 显示了该序列上的一 些跟踪结果. 很容易看出, 此融合跟踪方法是最好 的, 其原因是在多模态融合系统中, 即使某些特征 是无效的, 也总是有其他特征能够与模板匹配.

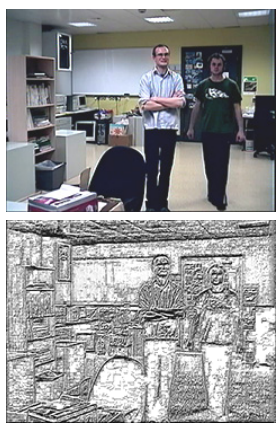

a. LBP

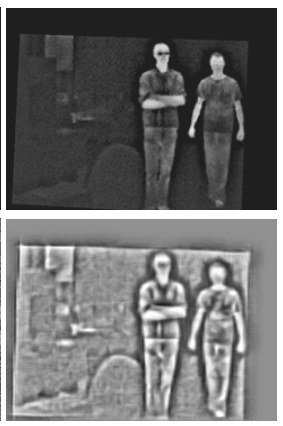

b. HOG
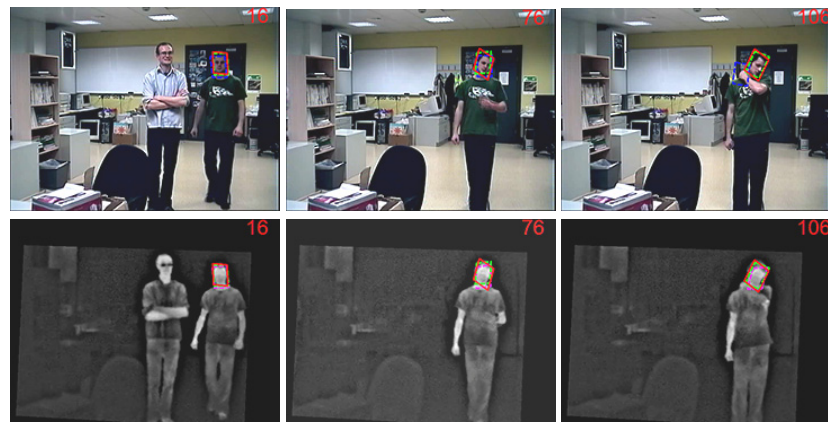

c. 关键帧跟踪结果的截图

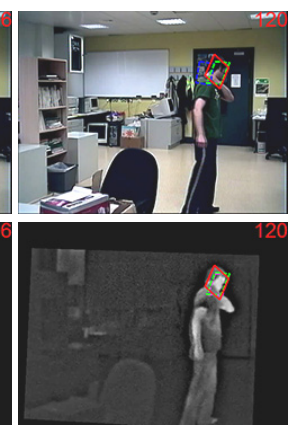

图 54 特征融合系统在图像序列 outrota 上的融合跟踪

\section{2 在单光谱序列上测评}

OTB50 和 VOT-TIR 是 2 个单模态数据集, 前 者包含 50 个可见光序列, 而后者包含 20 个红外序 列. 从 2 个数据集中各选择 4 个序列(详情请参阅 表 1), 并用灰度特征和 LBP 特征来描述可见光目 标、用灰色特征和 HOG 特征来描述红外目标. 图 6 分别绘制了 8 个序列的定位精度和成功率, 图例 上的数字代表位置误差國值为 20 个像素的定位精 度和重叠率阈值为 $50 \%$ 的成功率. 结果表明, 在 $\xi_{1}=20, \xi_{2}=0.5$ 时, 本文方法具有较高的精度, 且均优于 KCF 和 L1APG. 不难发现, 当重叠阈值 大于 0.75 时, 本文的跟踪器的成功率低于 L1APG, 这表明, 对于 MJCT 来说, 其处理目标尺度变化的 能力还有待提高. 图 7 显示了在 8 个序列上的一些 跟踪结果的截图, 也表明了 MJCT 能有效地处理大
的挑战, 包括尺寸变化、光照变化、背景杂波、遮 挡、形变和运动模糊.

\section{4 结 语}

本文提出了统一的多模态融合跟踪框架, 它 通过定制 DALM 实现, 称为 MJCT. 在该框架中, 可以自由添加、删除和自然集成不同类型的特征, 并通过联合压缩表示来进行自然融合. 由于压缩 表示减少了稀疏模板的维数, 这就大大加快了求 解 $\ell_{1}$ 最小化问题的速度. 此外, 协同模板更新策略 使跟踪器能够及时响应外观的变化. 11 个序列的 实验结果表明, 与目前最新的跟踪方法相比, 本文 方法取得了较好的性能, 但其处理完全遮挡能力 还有待提高.
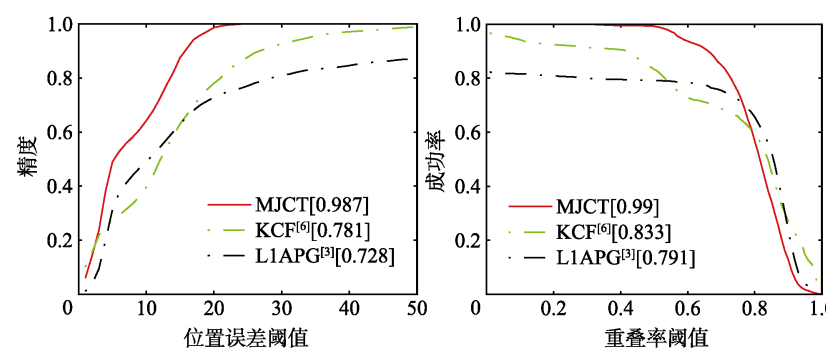

a. 可见光序列
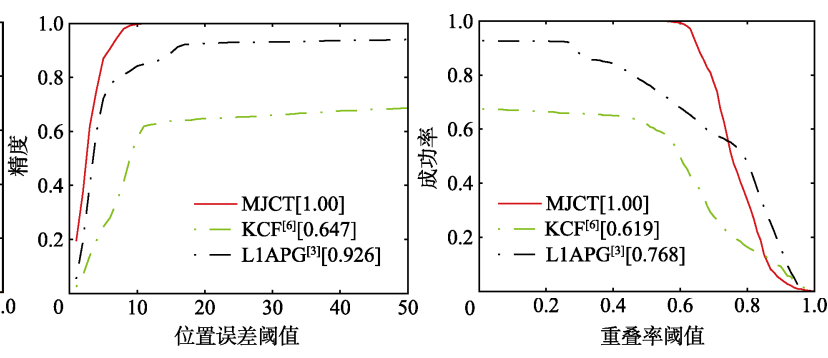

b. 红外序列

图 6 不同方法在后 8 个序列上跟踪的定位精度和成功率 


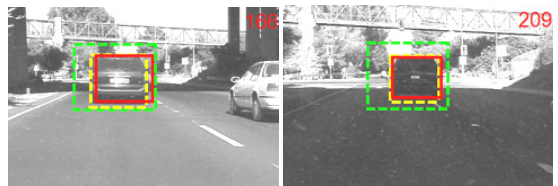

a. car4

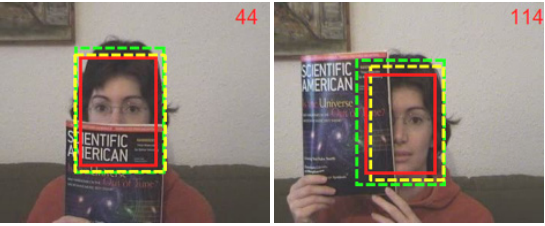

c. faceocc 1

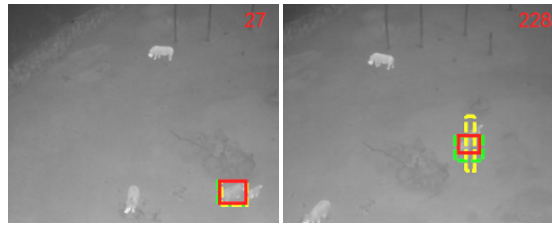

e. rhino

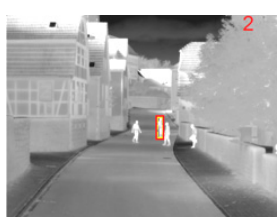

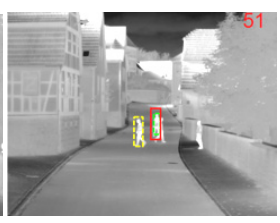

g. street
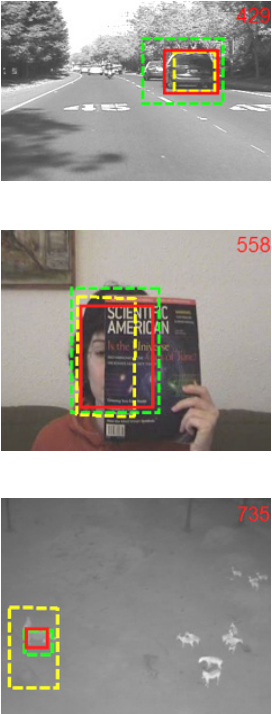

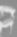
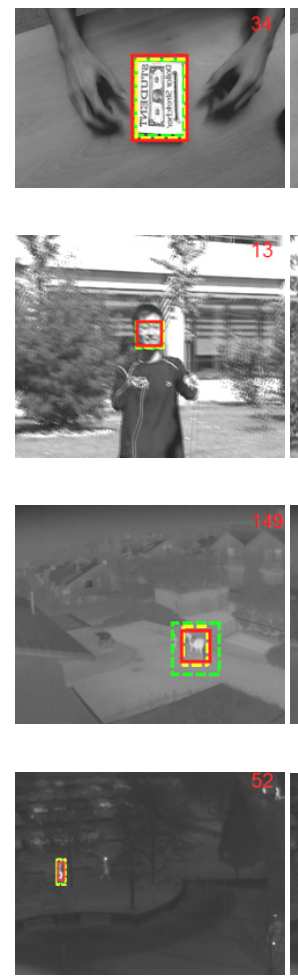

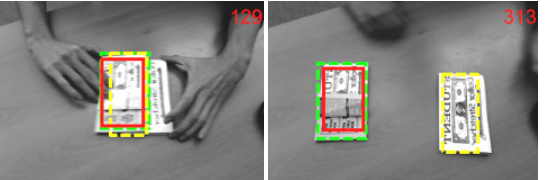

b. coupon

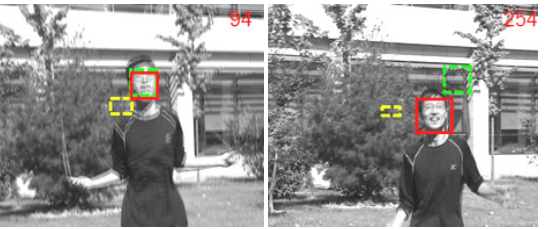

d. jump

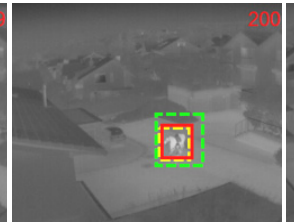

f. horse

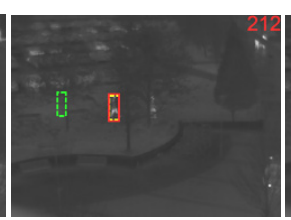

h. trees 1

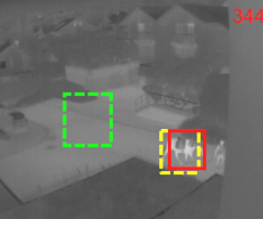

\section{$-\mathrm{MJCT}$ (本文); $\quad---\mathrm{KCF}^{[6]} ; \quad---\mathrm{L}^{\mathrm{APPG}}{ }^{[3]}$}

图 7 在后 8 个序列上跟踪结果的部分截图

\section{参考文献(References):}

[1] Zhang Canlong, Tang Yanping, Li Zhixin, et al. Dual-kernel tracking approach based on second-order spatiogram[J]. Journal of Electronics \& Information Technology, 2015, 37(7): 1660-1666(in Chinese)

(张灿龙, 唐艳平, 李志欣, 等. 基于二阶空间直方图的双核 跟踪算法[J]. 电子与信息学报, 2015, 37(7): 1660-1666)

[2] Mei X, Ling H B. Robust visual tracking using $\ell_{1}$ minimization[C] //Proceedings of the 12th IEEE Conference on Computer Vision. Los Alamitos: IEEE Computer Society Press, 2009: 1436-1443

[3] Bao C L, Wu Y, Ling H B, et al. Real time robust $\ell_{1}$ tracker using accelerated proximal gradient approach[C]//Proceedings of the IEEE Conference on Computer Vision and Pattern Recognition. Los Alamitos: IEEE Computer Society Press, 2012: 1830-1837

[4] Zhang T Z, Bibi A, Ghanem B. In defense of sparse tracking: circulant sparse tracker[C] //Proceedings of the IEEE Conference on Computer Vision and Pattern Recognition Los Alamitos: IEEE Computer Society Press, 2016: 3880-3888

[5] Gao J Y, Zhang T Z, Yang X S, et al. Deep relative tracking[J]. IEEE Transactions on Image Processing, 2017, 26(4): 1845-1858

[6] Henriques J F, Caseiro R, Martins P, et al. High-speed tracking with kernelized correlation filters[J]. IEEE Transactions on Pattern Analysis and Machine Intelligence, 2015, 37(3): 583-596
[7] Zoidi O, Tefas A, Pitas I. Visual object tracking based on local steering kernels and color histograms[J]. IEEE Transactions on Circuits System and Video Technology, 2013, 23(5): 870-882

[8] Lan X Y, Ma A J, Yuen P C. Multi-cue visual tracking using robust feature-level fusion based on joint sparse representation[C] // Proceedings of the IEEE Conference on Computer Vision and Pattern Recognition. Los Alamitos: IEEE Computer Society Press, 2014: 1194-1201

[9] Zhang L C, Danelljan M, Gonzalez-Garcia A, et al. Multi-modal fusion for end-to-end RGB-T tracking[C] //Proceedings of the IEEE International Conference on Computer Vision. Los Alamitos: IEEE Computer Society Press, 2019: 4612-4616

[10] Ma C, Liu C C, Peng F R, et al. Multi-feature hashing tracking[J]. Pattern Recognition Letters, 2016, 69(1): 62-71

[11] Jiang H L, Li J H, Wang D, et al. Multi-feature tracking via adaptive weights[J]. Neurocomputing, 2016, 207: 189-201

[12] Zhang X C, Ye P, Peng S Y, et al. SiamFT: an RGB-infrared fusion tracking method via fully convolutional siamese networks[J]. IEEE Access, 2019, 7: 122122-122133

[13] Zhang C L, Tang Y P, Li Z X, et al. Joint spatiograms for multi-modality tracking with online update[J]. Pattern Recognition Letters, 2019, 127: 128-137

[14] Xiao G, Yun X, Wu J M. A multi-cue mean-shift target tracking approach based on fuzzified region dynamic image fusion[J]. Science China Information Sciences, 2012, 55(3): 577-589

[15] Xiao G, Yun X, Wu J M. A new tracking approach for visible and infrared sequences based on tracking-before-fusion[J]. International Journal of Dynamics and Control, 2016, 4(1): 40-51 
[16] Yun X, Jing Z L, Xiao G, et al. A compressive tracking based on time-space Kalman fusion model[J]. Science China: Information Sciences, 2016, 59: 1-15

[17] Liu H P, Sun F C. Fusion tracking in color and infrared images using joint sparse representation[J]. Science China: Information Sciences, 2012, 55(3): 590-599

[18] Smeulders A W M, Chu D M, Cucchiara R, et al. Visual tracking: an experimental survey[J]. IEEE Transactions on Pattern Analysis and Machine Intelligence, 2013, 36(7): 1442-1468

[19] Wu Y, Lim J, Yang M H. Online object tracking: a benchmark[C]//Proceedings of the IEEE Conference on Computer Vision and Pattern Recognition. Los Alamitos: IEEE Computer Society Press, 2013: 2411-2418

[20] Yuan X T, Yan S C. Visual classification with multi-task joint sparse representation[J]. IEEE Transactions on Image Processing, 2012, 21(10): 4349-4360

[21] Li H X, Shen C H, Shi Q F. Real-time visual tracking using compressive sensing[C] //Proceedings of the IEEE Conference on Computer Vision and Pattern Recognition. Los Alamitos: IEEE Computer Society Press, 2011: 1305-1312

[22] Yang A Y, Zhou Z, Balasubramanian A G, et al. Fast $\ell_{1}$-minimization algorithms for face recognition[J]. IEEE Transactions on Image Processing, 2013, 22(8): 3234-3246

[23] Wright J, Yang A Y, Ganesh A, et al. Robust face recognition via sparse representation[J]. IEEE Transactions on Pattern Analysis and Machine Intelligence, 2009, 31(2): 210-227

[24] Achlioptas D. Database-friendly random projections: JohnsonLindenstrauss with binary coins[J]. Journal of Computer and System Sciences, 2003, 66(4): 671-687

[25] Dalal N, Triggs B. Histograms of oriented gradients for human detection[C] //Proceedings of the IEEE Conference on Computer Vision and Pattern Recognition. Los Alamitos: IEEE Computer Society Press, 2005: 886-893

[26] Vondrick C, Khosla A, Pirsiavash H, et al. Visualizing object detection features $[\mathrm{J}]$. International Journal of Computer Vision, 2016, 119(2): 145-158 